Andrzej Zieliński Uniwersytet Jagielloński andrzej.zielinski@uj.edu.pl

\title{
Relaciones deícticas espacio-temporales: análisis comparativo del sistema de los demostrativos polacos y españoles
}

\begin{abstract}
:
Spatio-Temporal Deixis Relationship. A Comparative Analysis of the Demonstrative Systems of Polish and Spanish

The main objective of this paper is to show the differences and similarities in the use of demonstrative forms in Polish and Spanish, which arise not only from differences paradigmatic (Spanish ternary system $v s$ Polish binary system), but also pragmatic context. With the help of electronic corpora the author tries to answer the question of what factors influence the conceptualization of these deictic expressions in both languages.
\end{abstract}

Keywords: deixis, demonstrative forms, space, time

\section{Streszczenie:}

Relacje deiksy czasoprzestrzennej. Analiza porównawcza systemu form wskazujących $w$ języku polskim i hiszpańskim

Głównym celem niniejszego studium jest ukazanie różnic i podobieństw w użyciu form wskazujących w języku polskim i hiszpańskim, które wynikają nie tylko z rozbieżności paradygmatycznych (hiszpański system 
trynarny $v s$ polski system binarny), ale i kontekstów pragmatycznych. Przy pomocy korpusów elektronicznych autor próbuje odpowiedzieć na pytanie, jakie czynniki wpływają na konceptualizację tych wyrażeń deiktycznych w obu językach.

Słowa kluczowe: deiksa, formy wskazujące, czas, przestrzeń.

\section{Introducció}

Como es bien sabido, en todas las lenguas nos encontramos con los elementos lingüísticos que solo pueden ser interpretados debidamente en un contexto determinado, ya que el significado que se desprende de ellos difiere mucho según una u otra situación contextual. Son las formas deícticas que sirven, grosso modo, de "vínculo referencial" [Eguren, 1999: 932] entre el significado que emana de ellas y su representación en el mundo real y social. Lyons [1977: 637-657], Lewinson [2006: 25-28] o Sosnowski [2010: 10], entre otros, hacen hincapié en que el punto de referencia, también denominado tradicionalmente origo o punto cero, de expresiones de la misma índole es el emisor, que conceptualiza el mundo rodeado desde una perspectiva egocéntrica (respecto a que YO estoy AQUÍ y AHORA).

Dentro de estas relaciones, se inscriben en ambas lenguas las formas demostrativas (zaimki wskazujące), cuyo objetivo principal es ubicar de la forma más precisa posible un objeto en el espacio o un acontecimiento en el eje temporal, lo cual constituye el tema del presente estudio. Como demostraremos más adelante, el emisor no siempre llega a codificarlo de forma precisa en ambas lenguas, debido, entre otras razones, a la gradación subjetiva que emana de ello. Así, sontotalmentegramaticales los siguientessintagmas:

(1) (pol.) Na tym świecie, na tej planecie, w tym państwie, w tym roku, w tym wieku.

(esp.) En este mundo, en este planeta, en este país, este año, en este siglo.

La selección del tema no ha sido casual, ya que, a pesar del creciente interés por la gramática contrastiva polaco-española que 
venimos observando en los últimos años, no hay estudios acerca de esta categoría tan esencial a la hora de codificar, bien el espacio por donde nos movemos, bien el eje temporal donde situamos el acontecimiento descrito. Se aparta de ello el ya esencial trabajo de Pawlik [2001: 99-101], donde el investigador dedica unas páginas a describir concisamente el análisis comparativo. Así pues, a fin de rellenar este espacio relativamente vacío, proponemos un estudio de los sistemas de los demostrativos en ambas lenguas, concentrándonos particularmente en los elementos distintivos que separan estos dos códigos lingüísticos. No es una cuestión baladí que los demostrativos sean los elementos que más problemas plantearán, tanto para los polacohablantes a la hora de aprender el español, como para los hispanohablantes en la misma situación de aprendizaje en lengua polaca. Debido al limitado espacio del que disponemos, nos ocuparemos únicamente del empleo espacio-temporal. Asimismo, partimos de la convicción de que tanto en polaco como en español estamos ante un sistema de demostrativos que abarca los empleos pronominal y adjetival sin que haya distinción gráfica entre ellos (véase el esquema 1). La principal dicotomía estriba, pues, en la función sintáctica que desempañan: sea el núcleo del SN \{(esp.) este (= el jersey) no me gusta; (pol.) ten (= sweter) nie podoba mi się\} sea su adyacente $\{(\mathrm{esp}$.$) este jersey no me gusta, (pol.) ten sweter nie po-$ doba mi się\}. De igual manera, en los dos paradigmas existen formas únicamente pronominales, de carácter neutro, invariables en número y género $\{(\mathrm{esp}$.$) esto, eso, aquello, (pol.) to, tamto \}$ que desempeñan una función presentadora:

\section{Pronombres demostrativos}

este, ese, aquel

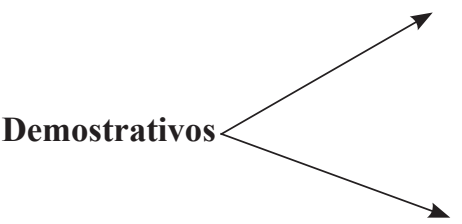

Zaimki rzeczowne wskazujące

ten, tamten

\section{Adjetivos demostrativos}

este, ese, aquel

Zaimki przymiotne wskazujące

Esquema 1

ten, tamten 
Por todo lo señalado, la estructura de nuestro trabajo se plasmará del siguiente modo: en primer lugar (2), abordaremos brevemente el sistema castellano: su morfología y funciones. Luego (3), pasaremos a analizar el sistema binario polaco con todas sus peculiaridades. Los datos emanados de los apartados anteriores nos permitirán percibir la dicotomía que separa estas lenguas, que presentaremos en forma de resumen en el último apartado (4).

\section{Sistema español de demostrativos}

El paradigma castellano está compuesto de tres elementos, que varían en número y género con el sustantivo al que complementan o sustituyen. Ese trinomio es una herencia del antiguo sistema latino que se mantiene en todas las lenguas iberorrománicas. Asimismo, la posición de los demostrativos españoles, en función de adyacente, puede verse alterada, dado que admiten empleos antepuestos y pospuestos. Como se observa en (2a), esta cubre principalmente valores de identificación y localización deíctica [Eguren, 1999: 950-951] de forma que no hace falta actualizar el SN por medio de otro actualizador explícito (*a la esta chica). En cambio, con la posposición del adjunto demostrativo (2b) el valor que resalta es únicamente la localización deíctica.

(2) a. ¿Tú qué le has hecho a esta chica? (C. Resino, Pop y patatas fritas, CREA)

b. Se lo decía antes a la chica esta (C. Martín Gaite, Fragmentos de interior, CREA)

Asimismo, la principal diferencia entre ambos empleos reside en el papel discursivo expresivo que desempeña el deíctico pospuesto. En este sentido, todo apunta a que la escisión de ambas nociones hace que el adjetivo demostrativo pospuesto llegue a señalar un mayor gra- 
do de implicación en la situación contextual en que se encuentra; con ello, de la implicación descrita pueden surgir diversas connotaciones semánticas, tales como: ese 'alejamiento psicológico' y, de ahí, 'desprecio' o este 'acercamiento valorativo', de donde surge ‘apreciación'. Así, en función a lo señalado, la posición pospuesta del demostrativo de carácter expresivo provoca su abrumadora frecuencia en el registro oral:

(3) a. me entretuve mucho en subirle la llave al hombre ese (C. Martín Gaite, Fragmentos de interior, CREA)

b. Y como tiene ya... y como ve la vida desde una larga experiencia todo lo observa con un carácter risueño, humorado, es fantástico el hombre este. (Hoy por hoy, 13/05/97, Cadena SER, CREA, registro oral)

Ahora bien, tradicionalmente se ha llegado a opinar que las tres formas deícticas están asignadas a las tres personas gramaticales que participan en el discurso, de tal forma que al yo le corresponde este, al ese le corresponde tú y el objeto de la comunicación (él se corresponde con aquel) [Alarcos Llorach, 1999; Pawlik, 2001: 94; entre otros]. Los ejemplos (4a-3c) ilustran debidamente esa relación espacial. Así, esta silla, del (3a), alude a la ubicación en las proximidades del emisor, esa pistola, del (3b), indica la localización cerca del interlocutor y aquella laguna, del (4c), no está al alcance de los participantes de la enunciación:

(4) a. Me quedo aquí contigo, bajo esta luz, en esta silla. (J. Díaz, Pablo Neruda viene volando, CREA)

b. Hijo, dame esa pistola. (Amestoy Egiguren, I. Ederra, CREA)

c. ¿No ven aquella laguna resplandeciente? (A. Sastre, El viaje infinito de Sancho Panza, CREA)

Por todo ello, en el caso de la deixis de tipo ad oculus, se ha propuesto la representación del sistema analizado en forma de círculos, de tal manera que a este le correspondería la cercanía del emisor (este = aqui), a ese el círculo cerca del destinatario (ahi) y, finalmente, a aquel le pertenecería otro círculo fuera del alcance de los dos (alli). Gráficamente podemos plasmarlo de modo siguiente: 

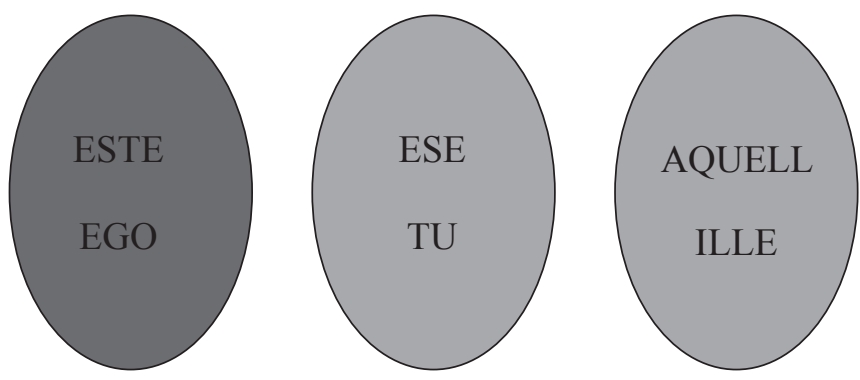

Esquema 2

Como pone de manifiesto Eguren [1999: 940], el sistema descrito no es perfecto funcionalmente, ya que la distinción entre los deícticos la establece, ante todo, el emisor, basándose en su previa experiencia espacio-temporal. Amén de ello, la distancia entre los círculos resulta poco nítida semántica y pragmáticamente, de ahí que el emisor pueda modificarlo por el proceso de subjetivización. Creemos que de todo ello se desprende el hecho de que el elemento más vago de los demostrativos en castellano resulta el de la distancia media, que puede llegar a neutralizarse a favor del primer elemento o del tercero, con tal de acercarlo o alejarlo respectivamente. En torno a la misma pista, la RAE [2010] parece afirmar que, en el español peninsular, el sistema etimológicamente ternario se va reduciendo paulatinamente al binario: el elemento ese tiende a desaparecer en las relaciones espaciales:

(5) Entonces, este, de ahí es que todo lo que aprendo. (B. Benítez de Lezcano, Encuesta, CREA, oral)

En este sentido, Kany [1994: 17-171] puso el dedo en la llaga al demostrar que en el español de América opera una clara neutralización entre los valores del segundo orden y del tercero a favor del segundo, de tal manera que el elemento aquel queda relegado, más bien, al registro literario.

En cuanto a su referencia temporal, el empleo de tres formas demostrativas cubre toda la distancia entre el AHORA y un acontecimiento de pasado o futuro. Ahora bien, el demostrativo de cercanía 
esta ubica un evento en el presente o en el momento de la enunciación y denota acciones pasadas o venideras:

(6) a. Esta semana he visitado a Paco y Lucía.

b. Ese mes fui a París.

c. Aquel año fue memorable.

Por lo general, ese alude a un evento anterior respecto al momento de enunciación, mientras que aquel denota una acción más remota. Sin embargo, el español actual tiende a reducir este sistema en su referencia temporal retrospectiva a dos elementos: este y aquel, que sustituye valores propios de ese [Eguren, 1999: 950-951; entre otros]. Lo podemos apreciar detenidamente en el ejemplo (6), donde la diferencia entre ese mes y aquel año es de carácter sumamente subjetivo, de ahí que resalte su valor poco nítido: en este caso, el emisor pretende subrayar algún vínculo con algún acontecimiento. Sin embargo, aquel queda restringido únicamente a la perspectiva retrospectiva, por lo que para referirse a un acontecimientovenidero cubre el empleo de la forma de la distancia media [RAE, 2010: 1280].

(7) a. Ese día ya lo verás.

b. *Aquel día ya lo verás.

\section{Sistema polaco de demostrativos}

A pesar de la opinión comúnmente aceptada, el sistema polaco consta únicamente de dos formas: ten 'este' y tamten 'aquel'. El elemento problemático ów,que algunos asocian, conforme a su etimología, con el elemento castellano de lejanía 'aquel', posee actualmente valores únicamente enfáticos de índole anafórica y resulta propio, más bien, del registro escrito (teniendo su correspondencia en el francés con voilà o en el latín con ECCE, [Kryk, 1987]) 
(8) Ów pan w końcu do mnie zadzwonił.

(Mismo señor finalmente a mí llamó)

'Aquel señor en persona vino a llamarme por teléfono'

Es más: los investigadores polacos insisten en que el elemento en cuestión es perfectamente intercambiable con otros elementos demostrativos (ów pan $=$ ten pan = tamten pan). Como es lógico, se sustrae de ello la función presentadora, incompatible con el elemento enfático:

(9) To jest moja szefowa.

* Owa jest moja szefowa.

El sistema polaco, de igual manera que en el sistema español, permite tanto la anteposición como la posposición del adyacente del núcleo del SN. Sin embargo, en tal caso no operan el acercamiento o el alejamiento pragmáticos, como observamos en español, sino que adquiere únicamente su función anafórica, como podemos ver en un típico encabezamiento de cuentos infantiles:

(10) a. Był sobie król. Król ten miał córkę...(Érase rey. Este rey tenía hija) 'Erase una vez un rey. Este rey tenía una hija'

b. *Był sobie król. Król miał córkę.

(Érase rey. Rey tenía hija)

Además, como se desprende debidamente del ejemplo (10b), la posposición del adjetivo demostrativo se hace obligatoria en la lengua polaca cuando el sustantivo aparece expreso previamente en el mismo discurso. Así, en (11) vemos que el sintagma nominal kobieta ta ('la mujer esta') alude al sustantivo Żydówka ('judía'), que aparece al inicio de la enunciación. De hecho, su omisión hace que la oración sea totalmente agramatical [Pisarkowa, 1969: 52; Kryg, 1987].

(11) Żydówka, o której doktor jeszcze po latach nie mógł mówić spokojnie.

$\mathrm{O}$ ile się orientowałem, poszlaki były słabe i wielu uważało, że kobieta ta z całą pewnością była niewinna. (A. Bolecka, Uwiedzeni, NKJP)

En torno a la misma pista, notamos que el mismo valor anafórico -eso sí, menos enfático- deviene de la anteposición del mismo 
elemento en el SN polaco. Apurando un poco más, veremos que el demostrativo de primer orden polaco, por su relativa alta frecuencia en el polaco actual, llega a tener una noción puramente actualizadora y delimitadora, de ahí que Pisarkowa [1969: 51-58] se decantara por marcarlo como elemento delimitador. En este sentido, libres de prejuicios lingüísticos, apreciamos que su abrumadora frecuencia en el registro coloquial incide en el hecho de que pueda llegar a gramaticalizarse en el transcurso del tiempo como un elemento estrictamente gramatical, aunque creemos que de su empleo todavía deriva la función de deixis ad phantasma, como veremos en el ejemplo de abajo. Siguiendo esta línea, creemos preciso añadir que Kany [1994: 171172] observa algo parecido en el español de América, denominándolo "este de relleno":

(12) Ale to ci mówię, to no to sześć talerzyków płaskich, sześć tych wszystkich, cukierniczka, dzbanek i te wszystkie dodatkowe rzeczy, wiesz, takie te te na te na no tam parę tych elementów, nie. (Rozmowa o jedzeniu, NKJP)

(pero esto te lo digo, esto esto seis platitos, seis estos todos, azucarero, jarrón, y estas todas cosas adicionales, estas estas sobre estas sobre [...] ahí)

En todo caso, como ya hemos indicado, a diferencia del sistema castellano, el paradigma de los demostrativos polaco es binario: consta de dos elementos deícticos - ten y tamten - que concuerdan en número, género y caso con el sustantivo. Como es de suponer, de la diferencia cuantitativa emana la dicotomía cualitativa, lo cual vamos a demostrar a continuación.

Desde la perspectiva espacial, observamos que en la función de deixis de tipo ad oculos, el elemento de primer orden ten alude a la cercanía del emisor, en cambio, el demostrativo tamten engloba su localización de lejanía. En opinión de Klemensiewicz [1986: 56], es la típica situación referencial cuando el emisor indica un objeto con un gesto: 
(13) a. Daj mi tę książkę.

(dame este libro)

b. Pożycz mi tamten długopis.

(préstame aquel bolígrafo)

En este sentido, los ejemplos de arriba demuestran claramente que su empleo no tiene nada que ver con las personas que participan en el discurso, pese a la opinión contraria de Kryk [1987]. Esto es así, dado que es el emisor parte específicamente de su experiencia espacial y, por consiguiente, es el que determina en gran medida su concepción en el espacio. Así, gráficamente podemos plasmarlo en forma de un círculo alrededor del yo del enunciado:

Esquema 3

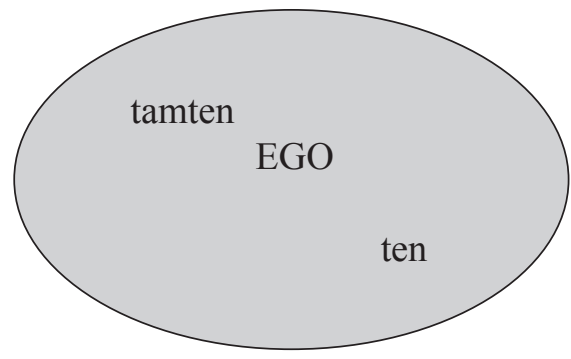

Debido a la imprecisión semántica entre los dos deícticos, el elemento de cercanía empieza a usurpar valores antiguamente reservados a tamten, de tal manera que se lleva a cabo una clara neutralización entre ambos a favor del primero [Pisarkowa, 1969; Kryk, 1987]. Creemos que dicho proceso está motivado por la subjetivización que opera en los niveles semántico y pragmático, insertando el elemento subjetivo en el enunciado. Por consiguiente, el elemento ten adquiere más fuerza ilocutiva. Veamos algunos ejemplos:

(14) a. Za tobą jest ten profesor (o którym ci mówiłem).

(Detrás de ti está este profesor (del que te he hablado))

b. (??) Za tobą jest tamten profesor (o którym ci mówiłem).

(Detrás de ti está aquel profesor (del que te he hablado)) 
Para un polacohablante la oración (14a) resulta gramatical, aunque poco precisa por el requerimiento de un contexto más específico; en cambio, la (14b) parece totalmente anormal, no sólo por la misma falta, sino también por la ausencia de la referencia fórica, de ahí que el enunciado resulte incomprensible para cualquier interlocutor. $\mathrm{Y}$ aunque se introduzca el contexto explícito, el contenido no queda del todo claro.

(15) Podaj mi nożyczki. Nie te, tamte!

(Pásame las tijeras. ¡No estas, aquellas!)

Así pues, como una clara consecuencia de la neutralización de valores deícticos antes comentada, se observa en el polaco actual una restricción del empleo de la forma de lejanía. A saber: aparece particularmente en contraste con el primer elemento [Pisarkowa, 1969: 60] y, por lo tanto, el deíctico ten se convierte en el punto de referencia para el demostrativo tamten. Eso, a su vez, nos permite entender la total incomprensividad del ejemplo (14b). Con todo, nuestra investigación del NKJP abunda en ejemplos:

(16) a. Nie da się nie widzieć, że mieszkała któraś w tym domku, teraz mieszka w tamtym.

(No se puede no ver que vivió alguna en esta casa, ahora vive en aquella...)

'Se puede ver que alguien vivió en esta casa, aunque ahora vive en quella... (W. Myśliwski, Traktat o tuskaniu fasoli, NKJP)

b. Moi panowie - ozwał się nagle, zwracając głowę ku tamtym.

(Mis señores - respondió de repente, volviendo la cabeza hacia aquellos) (L. Kruczkowski, Kordian i cham, NKJP)

Para la referencia temporal (caso de la deixis am phantasma) observamos la misma restricción: si el contexto en que aparece el demostrativo está claramente delimitado, entonces lo normal es que se emplee el elemento de primer orden, aunque la referencia temporal aluda a una acción remota, acabada: 
(17) a. na Wschodnim Wybrzeżu było w tym momencie w powietrzu przynajmniej około tysiąca samolotów pasażerskich. (A. Barczyński, Ślepy los, NKJP)

(en la Costa Oriental hubo en este momento en el aire aprox. mil aviones de pasajeros)

b. W tym czasie nie było prądu.

(En este tiempo no había electricidad)

'En aquel tiempo no había electricidad'

Amén de ello, el empleo del demostrativo de lejanía en la referencia temporal provoca ad hoc la lectura referencial en el momento de enunciación, su origo (conforme con que YO estoy AQUÍ y AHORA) Así, al enunciar (18), el emisor ubica el acontecimiento descrito antes del momento de enunciación, de ahí que el interlocutor lo interprete como 'el anterior al presente', es decir 'el año pasado':

(18) W tamtym roku byłem w Peru.

(en aquel año estuve en Perú)

'estuve en Perú el año pasado'

La búsqueda en el corpus electrónico del NKJP no hace más que confirmarlo. Así, se puede observar en (19) que el complemento circunstancial de tiempo $w$ tym momencie ('en aquel momento') alude a un acontecimiento anterior al momento en el que el narrador se decide a contárnoslo:

(19) Oczywiście nie miałem żadnej [broni], jednak w tamtym momencie była to pierwsza rzecz, jaka przyszła mi na myś. (D. Zieliński, Manuskrypty, NKJP)

([...] Por supuesto, no tenía ninguno [arma], pero en aquel momento era la primera cosa que se me vino a la cabeza)

Además de todo lo señalado, el deíctico ten es el único que empleamos para referirnos a un evento venidero. Nos inclinamos a pensar que la selección de ten se debe a la perspectiva egocéntrica (YO - EN - MOVIMIENTO) según la cual estamos orientados de cara hacia el futuro, de ahí que sea concebido como el elemento más cercano: 
(20) Kryzys skończy się za kilka lat, może szybciej. Dopiero ten moment będzie prawdziwym Testem [...]. (M. Góra, "Jak nie kijem, to i nie pałką", Polityka, NKJP)

(La crisis terminará dentro de unos años, quizás antes. Este momento será una verdadera prueba $[\ldots]$ )

Vemos, pues, que de estos dos elementos el primero cubre un campo de empleo mayor, tanto para la referencia espacial como temporal [Pawlik, 2001: 99; Kryk, 1987: 46-48]. Esto es así, ya que el elemento tamten, semántica y pragmáticamente, resulta periférico: exige un punto de referencia espacio-temporal, enmarcado en $t u$, sea explícito sea implícito,al que debe contrastar, expresando así su respectiva lejanía.

\section{Resumen}

Como ya dijimos al inicio del presente artículo, el español consta de un sistema ternario y el polaco de uno binario. De la diferencia cuantitativa resalta la cualitativa. Así, en primer lugar, la organización del sistema de demostrativos castellano se basa, grosso modo, en la localización de objetos o eventos más o menos precisa en el eje espacio-temporal con respecto al punto de referencia del emisor, que se funda en su experiencia previa en estos dominios. En cambio, el sistema polaco se fundamenta en la oposición de dos deícticos, de los cuales el primero, no marcado, usurpa empleo mayoritario, de ahí que le corresponda fácilmente el empleo de los tres deícticos castellanos, tanto en la referencia espacial como temporal.

(21) a) Aquel/ Ese hombre vino en aquel/ ese momento [...].

Ten czlowiek przyszedł $\mathrm{w}$ tym momencie [...].

b) Pásame aquel libro que está detrás de la mesa.

Podaj mi tę książkę, która jest za stołem.

c) Dame ese teléfono que tienes en el bolsillo.

Daj mi ten telefon, który masz w kieszeni. 
d) En aquel/ese año memorable nos casamos.

W tym pamiętnym roku wzięliśmy ślub.

El demostrativo tamten, en cambio, parece reservado únicamente a empleos que contrastan con ten, su punto de referencia, mencionado, bien explícita, bien implícitamente en el discurso. En tal caso, su función contrastiva corresponde en la lengua española a ese o aquel, según el contexto propiciado o necesidades pragmáticas del emisor:

(22) a) Dziecko płacze, bo nie chce tej zabawki, ale tamtą.

El niño está llorando, porque no quiere este juguete, pero (sino) aquel/ ese.

b) Dzisiaj trudno sobie wyobrazić, że w tamtych czasach nie było Internetu.

Hoy cuesta imaginar que en aquellos tiempos no hubiera Internet.

Asimismo, como clara consecuencia de la referencia subjetiva, la lectura de la posposición del demostrativo castellano adquiere nociones valorativas de alejamiento o acercamiento, inexistentes en polaco.

Para concluir, Nowikow [2003: 63] ya indicó que, desde una perspectiva tipológica, el polaco y el español -a pesar de ser ambas lenguas flexivas- pertenecen a dos grupos lingüísticos distintos: el polaco correspondería al tipo AC (aspecto + caso) y el castellano al Art.T. (artículo + sistema verbal más desarrollado). Los datos proporcionados por este estudio contrastivo apuntan al hecho de que a esta división habría que agregar, quizás, el funcionamiento de los demostrativos, puesto que la lengua española, según hemos demostrado, posee elementos más precisos que el polaco para ubicar un objeto o un acontecimiento en el espacio.

\section{Corpora}

CREA $=$ Corpus de Referencia del Español Actual, [on-line] www.rae.es 10.10.2012.

NKJP = Narodowy Korpus Języka Polskiego, [on-line] http://nkjp.pl/ 10.10.2012. 


\section{Bibliografía}

ALARCOS LLORACH, E. (1999), Gramática de la lengua española, Espasa Calpe, Madrid.

ÁLVAREZ MARTÍNEZ, M.A. (1989), El pronombre, Arco/Libros, Madrid. BORYŚ, W. (2005), Słownik etymologiczny języka polskiego,WL, Kraków. EGUREN, L. (1999), "Pronombres y adverbios demostrativos. Las relaciones deícticas", en: Bosque, I., Demonte, V. (eds.), Gramática descriptiva de la lengua española, vol. I, Espasa Calpe, Madrid, pp. 929-972.

HEINE, B. (1997), Cognitive Foundations of Grammar, Oxford University Press, Oxford.

JUNGBLUTH, K. (2001), "Binary and ternary deictic systems in speech and writing. Evidence from the use of demonstratives in Spanish", PhiN, 15, pp. 1-24.

KANY, Ch. (1994), Sintaxis hispanoamericana, Gredos, Madrid.

KLEMENSIEWICZ, Z. (1986), Podstawowe wiadomosći z gramatyki języka polskiego, $\mathrm{PWN}$, Warszawa.

KRYK, B. (1987), On deixis in English and Polish: the role of demonstrative pronouns, Verlag Peter Lang, Frankfurt am Main.

LEWINSON, S. (2006), Space in Language and Cognition, Cambridge University Press, Cambridge.

LYONS, J. (1977), Semantics, 2 vols, Cambridge University Press, Cambridge.

NOWIKOW, W. (2003), “Acerca de las relaciones entre el aspecto y el modo accional desde una perspectiva tipológico- comparada", Language Design, 5, pp. 51-66.

PAWLIK, J. (2001), Selección de problemas de gramática española, Wydawnictwo Naukowe UAM, Poznań.

PIĘTKOWA, R. (1989), Funkcje wyrażeń werbalizujących kategorie przestrzenne: na materiale wspótczesnej poezji polskiej, Wydawnictwo Uniwersytetu Śląskiego, Katowice.

PISARKOWA, K. (1969), Funkcje składniowe polskich zaimków odmiennych, PAN, Wrocław.

RAE (2010), Nueva gramática de la lengua española, 2 vols, Espasa, Madrid.

SOSNOWSKI, R. (2010), Deissi spaziale nei testi teatrali italiani del XVI secolo, Wydawnictwo UJ, Kraków. 